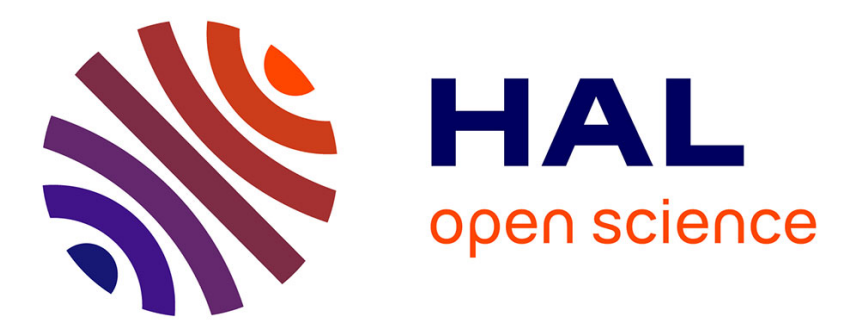

\title{
Réalisation d'un montage intégrateur destiné à la mesure du moment magnétique d'un supraconducteur de faible volume
}

\author{
J.-P. Brisset, J.-P. Girard, E. Paumier, J. Provost
}

\section{To cite this version:}

J.-P. Brisset, J.-P. Girard, E. Paumier, J. Provost. Réalisation d'un montage intégrateur destiné à la mesure du moment magnétique d'un supraconducteur de faible volume. Revue de Physique Appliquée, 1974, 9 (2), pp.497-499. 10.1051/rphysap:0197400902049700 . jpa-00243808

HAL Id: jpa-00243808 https://hal.science/jpa-00243808

Submitted on 1 Jan 1974

HAL is a multi-disciplinary open access archive for the deposit and dissemination of scientific research documents, whether they are published or not. The documents may come from teaching and research institutions in France or abroad, or from public or private research centers.
L'archive ouverte pluridisciplinaire $\mathbf{H A L}$, est destinée au dépôt et à la diffusion de documents scientifiques de niveau recherche, publiés ou non, émanant des établissements d'enseignement et de recherche français ou étrangers, des laboratoires publics ou privés. 


\author{
Classification \\ Physics Abstracts \\ $0.660-0.665-8.460$
}

\title{
RÉALISATION D’UN MONTAGE INTÉGRATEUR DESTINÉ A LA MESURE DU MOMENT MAGNÉTIQUE D'UN SUPRACONDUCTEUR DE FAIBLE VOLUME
}

\author{
J.-P. BRISSET, J.-P. GIRARD, E. PAUMIER et J. PROVOST \\ Laboratoire de Physique du Solide de l'Université de Caen, 14032 Caen-Cedex, France
}

(Reçu le $1^{\text {er }}$ août 1973)

\begin{abstract}
Résumé. - On décrit un montage utilisé pour mesurer le moment magnétique de petits échantillons supraconducteurs et on en discute la sensibilité. On donne un exemple d'application : l'enregistrement d'une courbe d'aimantation d'un disque mince de plomb.
\end{abstract}

Abstract. - A device used for measuring the magnetic moment of small superconducting samples is described and its sensitivity is discussed. The magnetization curve of a thin lead disc is given as an example.

1. Introduction. - L'intégration analogique est un procédé classique pour obtenir des informations sur l'état magnétique d'un échantillon plongé dans un champ extérieur [1], [2]. Elle consiste à intégrer le signal apparaissant aux bornes d'une bobine entourant l'échantillon, lorsqu'on fait varier de façon lente et continue le champ magnétique appliqué. Cette méthode, dans laquelle l'échantillon reste, en principe, constamment en équilibre est particulièrement adaptée au relevé des courbes d'aimantation des supraconducteurs. Sa principale difficulté est sa grande sensibilité aux signaux parasites d'origines diverses, surtout en champ appliqué faible. Nemoz et Coll. [2] ont proposé un intégrateur analogique permettant des intégrations d'une durée de l'ordre de 10 min., avec des dérives de l'ordre de quelques $\mathrm{nV}$.

A partir de ce principe, nous avons mis au point un montage permettant d'intégrer pendant 10 à 30 min. un signal de l'ordre du $\mu \mathrm{V}$, pour une tension de dérive ramenée à l'entrée de l'ordre de $2 \mathrm{nV}$.

2. Réalisation. - Le schéma de principe (Fig. 1) est inspiré de celui de $\mathrm{Nemoz}$ et Coll. [2].

Le premier étage, amplificateur, est constitué par un amplificateur modulaire, stabilisé par chopper, Analog-Devices type $232 \mathrm{~K}$. Ce module présente une dérive de tension de déséquilibre de l'ordre de $1 \mu \mathrm{V} / \mathrm{mois}$ et $0,05 \mu \mathrm{V} /{ }^{\circ} \mathrm{C}$. La dérive du courant de polarisation d'entrée, de l'ordre de $0,5 \mathrm{pA} /{ }^{\circ} \mathrm{C}$ a un effet négligeable, ainsi qu'on le verra au paragraphe 3 .

Cet amplificateur, de gain très élevé $\left(G=10^{7}\right)$ est associé à une résistance de contre-réaction $\mathrm{RCL}$, très stable en température $\left(\frac{1}{R} \frac{\Delta R}{\Delta T}=10 \times 10^{-6} /{ }^{\circ} \mathrm{C}\right)$.

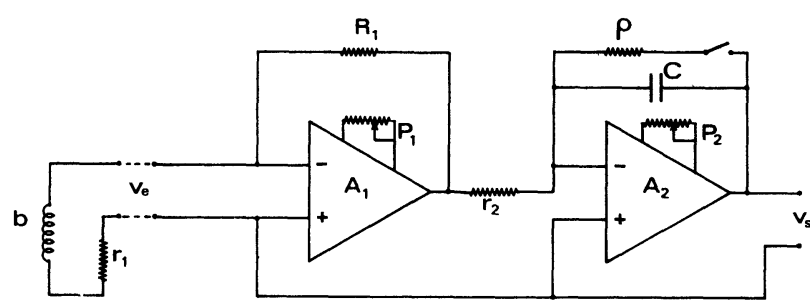

FIG. 1. - Schéma de l'amplificateur intégrateur. $b$ solénoïde de mesure $; r_{1} 260,8 \pm 0,2 \Omega ; R_{1} 1 \mathrm{M} \Omega 0,1 \% ; r_{2} 1 \mathrm{M} \Omega 0,5 \%$; $C 0,980 \pm 0,05 \mu \mathrm{F}, \rho 1 \mathrm{k} \Omega, P_{1} 100 \mathrm{k} \Omega 10 \mathrm{t}, P_{2} 500 \Omega 10 \mathrm{t}$, $A_{1}$ amplificateur Analog-Devices $232 \mathrm{~K}, A_{2}$ amplificateur Tranchant-Electronique TB $62 \mathrm{~B}$.

La résistance de source du montage est celle de la bobine de mesure qui, plongée dans l'hélium liquide est, aussi, parfaitement stable. Le gain réel est alors de l'ordre de 4000 .

Le second étage, intégrateur, est un amplificateur modulaire Tranchant TB $62 \mathrm{~B}$, choisi pour sa grande impédance d'entrée différentielle $\left(Z_{\mathrm{d}}=10^{11} \Omega\right)$. Il est associé à une capacité au parylène KEMET, choisie pour la qualité de son isolement (constante de temps de $10^{6} \mathrm{~s}$ ), et à une résistance RCL de $1 \mathrm{M} \Omega$.

Des essais ont montré qu'il n'est pas nécessaire de thermostater le montage, mais il est enfermé dans une enceinte en aluminium, à parois épaisses de $1 \mathrm{~cm}$, et de petites dimensions : l'ensemble mesure $10 \times 12 \times 6 \mathrm{~cm}$, sans son alimentation. Cette enceinte assure à la fois le blindage électrique et une bonne homogénéité de température. Il est certain que cette miniaturisation, ainsi que l'absence de tout commutateur dans l'étage d'entrée entre pour une bonne part dans les performances obtenues. 
En pratique, la liaison entre l'étage amplificateur et l'étage intégrateur est assurée par des microfiches démontables SODERN MF 5 pour thermocouple. Ceci permet de corriger en l'absence de signal, et séparément, les tensions de déséquilibre des deux étages. D'autre part, la bobine de mesure est reliée à l'entrée de l'étage amplificateur par l'intermédiaire d'un fil de cuivre allié et argenté FILOTEX 1875 qui, outre la qualité de résistance de son isolement aux chocs thermiques, ne présente qu'un effet Thomson très faible et très stable entre 4,2 et $300 \mathrm{~K}$. Ce fil est sorti sans solution de continuité depuis le bain d'hélium jusqu'à l'entrée de l'amplificateur, où il est connecté par l'intermédiaire de microfiches dorées AMP, serties sur le fil et blindées thermiquement.

3. Sensibilité en tension. - Les précautions énumérées étant prises, l'intégration d'un signal est possible à condition que la dérive propre de l'amplificateur soit suffisamment faible :

On sait que, le gain en boucle ouverte et la résistance d'entrée de l'amplificateur opérationnel étant suffisants, on peut écrire la tension de sortie du premier étage sous la forme :

$$
V_{\mathrm{s}}=-\frac{R_{1}}{r_{1}} V_{\mathrm{e}}-R_{1}\left(i_{\mathrm{d}}-\frac{V_{\mathrm{d}}}{r_{1}}\right)
$$

$V_{\mathrm{e}}$ est la tension appliquée à l'entrée, $r_{1}$ la résistance de source, $R_{1}$ la résistance de contre-réaction, $i_{\mathrm{d}}$ est le courant de polarisation et $V_{\mathrm{d}}$ la tension de déséquilibre.

L'appareil étant compensé au départ, c'est-à-dire le second terme de $V_{\mathrm{s}}$ annulé, on aura linéarité de l'amplification si ce second terme reste négligeable, soit :

$$
\Delta V_{\mathrm{d}} \ll V_{\mathrm{e}} \quad \text { et } \quad \Delta i_{\mathrm{d}} \ll \frac{V_{\mathrm{e}}}{r_{1}} \text { pendant la mesure } .
$$

Dans le cas du $232 \mathrm{~K}$, la première condition est très largement remplie pour $V_{\mathrm{e}}>1 \mu \mathrm{V}$, en ambiance thermique stable. La seconde condition est pratiquement toujours remplie. Le second étage est alors attaqué par une tension suffisante pour que les problèmes de dérive de cet étage soient secondaires.

4. Mise en œuvre. - Le capteur est constitué par un solénoïde de fil de cuivre, comportant $N$ spires par unité de longueur, compensé à ses extrémités par deux bobines enroulées en sens inverse du bobinage principal et mises en série avec lui, de telle sorte que, en l'absence d'échantillon, l'ensemble présente une f.e.m. nulle en champ variable, et se comporte comme un solénoïde infini vis-à-vis des dimensions de l'échantillon.

Imaginons que le champ appliqué $\mathbf{H}_{\mathbf{a}}$ soit produit en faisant circuler un courant $I$ dans la bobine de mesure. L'énergie d'aimantation de l'échantillon s'écrit alors :

$$
\delta W=\mu_{0} \int_{v} \mathbf{H}_{\mathrm{a}}(\mathbf{r}) . \delta \mathbf{J}(\mathbf{r}) \mathrm{d}^{3} r=I \dot{\delta} \varphi
$$

où $v$ est le volume de l'échantillon, $\mathbf{J}(\mathbf{r})$ l'intensité d'aimantation et $\delta \varphi$ la variation du flux d'induction créé par l'aimantation de l'échantillon dans le solénoïde de mesure. $\mathbf{H}_{\mathrm{a}}$ étant uniforme sur le volume de l'échantillon dont la forme est telle que $\mathbf{M}$ soit parallèle à $\mathbf{H}_{\mathrm{a}}$ on a alors :

$$
\delta W=\mu_{0} \mathbf{H}_{\mathrm{a}} \delta M=\mu_{0} N I \delta M=I \delta \varphi
$$

soit :

$$
\frac{\mathrm{d} \varphi}{\mathrm{d} t}=\mu_{0} N \frac{\mathrm{d} M}{\mathrm{~d} t}=V_{\mathrm{e}}
$$

tension aux bornes de la bobine.

Nous avons donc montré que dans ces conditions l'intégration du signal apparu aux bornes de la bobine de mesure donne directement le moment magnétique total de l'échantillon.

5. Possibilités du montage. - Nous avons vu que la tension minimale intégrable est de l'ordre de $1 \mu \mathrm{V}$. On a alors :

$$
V_{\mathrm{e}}=N \mu_{0} \frac{\mathrm{d} M}{\mathrm{~d} t}=10^{-6} \mathrm{~V}
$$

Dans notre dispositif, $N=3 \times 10^{5} \mathrm{trs} / \mathrm{m}$, la vitesse de balayage en champ est environ de $1 \mathrm{Oe} / \mathrm{s}$ $(79,56 \mathrm{~A} / \mathrm{m} / \mathrm{s})$ et le temps d'intégration supérieur à $600 \mathrm{~s}$, ce qui donne :

$$
M_{\min } \simeq 1,5 \times 10^{-3} \mathrm{~A} \cdot \mathrm{m}^{2} .
$$

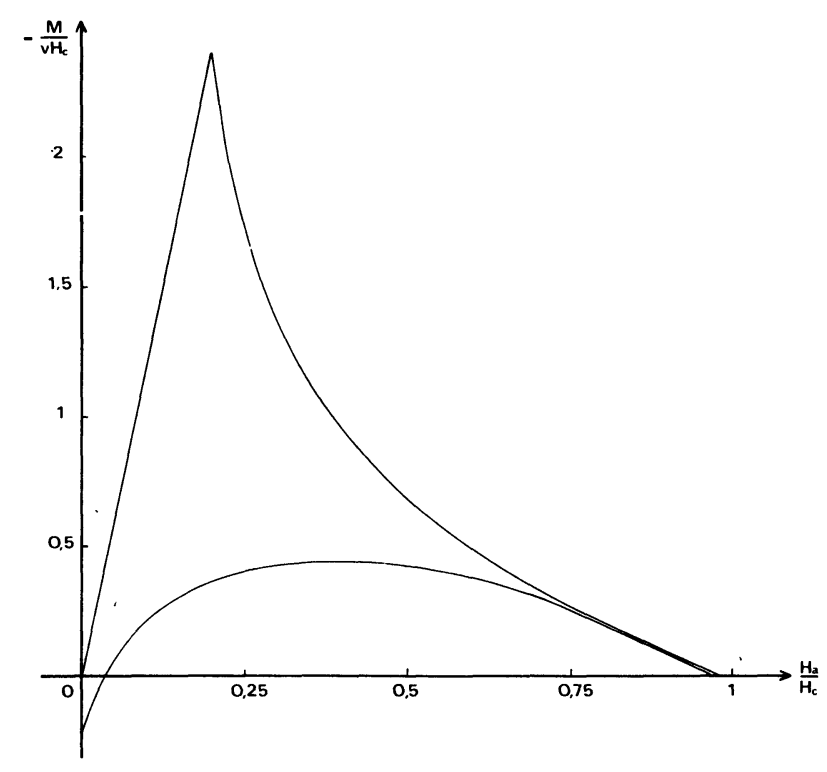

FIG. 2. - Courbe de première aimantation à $4,2 \mathrm{~K}$ d'un disque de plomb pur (diamètre $9 \mathrm{~mm}$, épaisseur $300 \mu \mathrm{m}$ ) en champ parallèle à l'axe. 
Pour un échantillon de plomb au voisinage du champ critique ceci correspond à un volume $v=M / J \simeq 30 \mathrm{~mm}^{3}$, soit une épaisseur de $300 \mu \mathrm{m}$ pour un disque de $100 \mathrm{~mm}^{2}$ de surface : on peut donc s'attendre à pouvoir relever des courbes d'aimantation exploitables jusque dans ce domaine d'épaisseur pour la dimension de disque utilisée ici.
6. Résultats. - La figure 2 montre la courbe d'aimantation à $4,2 \mathrm{~K}$ d'un disque de plomb de $9 \mathrm{~mm}$ de diamètre et $300 \mu \mathrm{m}$ d'épaisseur. La dérive enregistrée correspond au plus à une tension d'entrée moyenne de $2 \mathrm{nV}$ pour une durée d'intégration de 20 min., ce qui est bien conforme au résultat attendu.

\section{Bibliographie}

[1] Mc Evoy, J. P., Jones, D. P. and Park, J. G., Solid State Commun. 5 (1967) 641.
[2] Nemoz, A., Solecki, J.-C., Faure, J.-P. et Keyston, J. R. G., Revue Phys. Appl. 4 (1969) 42. 